\title{
Enhancement of plant cold tolerance by soybean RCC1 family gene GmTCF1a
}

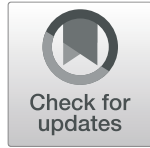

\author{
Zhanghui Dong ${ }^{1}$, Hui Wang ${ }^{2}$, Xia Li ${ }^{2}$ and Hongtao $\mathrm{Ji}^{2^{*}}$
}

\begin{abstract}
Background: Low temperature severely limits the growth, yield, and geographic distributions of soybean. Soybean plants respond to cold stress by reprogramming the expression of a series of cold-responsive genes. However, the intrinsic mechanism underlying cold-stress tolerance in soybean remains unclear. A. thaliana tolerant to chilling and freezing 1 (AtTCF1) is a regulator of chromosome condensation 1 (RCC1) family protein and regulates freezing tolerance through an independent C-repeat binding transcription factor (CBF) signaling pathway.

Results: In this study, we identified a homologous gene of AtTCF1 in soybean (named GmTCF1a), which mediates plant tolerance to low temperature. Like AtTCF1, GmTCF1a contains five RCC1 domains and is located in the nucleus. GmTCF1a is strongly and specifically induced by cold stress. Interestingly, ectopic overexpression of GmTCF1a in Arabidopsis greatly increased plant survival rate and decreased electrolyte leakage under freezing stress. A cold-responsive gene, COR15a, was highly induced in the GmTCF1a-overexpressing transgenic lines.

Conclusions: GmTCF1a responded specifically to cold stress, and ectopic expression of GmTCF1a enhanced cold tolerance and upregulated COR15a levels. These results indicate that GmTCF1a positively regulates cold tolerance in soybean and may provide novel insights into genetic improvement of cold tolerance in crops.
\end{abstract}

Keywords: Soybean, Cold tolerance, RCC1-like protein, AtTCF1, GmTCF1a

\section{Background}

As an important cash crop, soybean (G. $\max$ ) provides us not only abundant proteins but also oils for consumption. Soybean is a temperate legume and plants are particularly susceptible to low temperature stress, which significantly limits the growth of soybean and thus severely reduces yield [1]. Soybean seeds and germinating seedlings are extremely sensitive to low temperatures. During the seed germination stage, low temperature affects the germination of soybean seeds and reduces seedling emergence in cold soils by interfering with the normal membrane reorganization during seed imbibition [2]. Low temperatures of about $10^{\circ} \mathrm{C}$ or less sustained

\footnotetext{
* Correspondence: htji@mail.hzau.edu.cn

${ }^{2}$ National Key Laboratory of Crop Genetic Improvement, College of Plant Science and Technology, Huazhong Agricultural University, Wuhan 430070, China

Full list of author information is available at the end of the article
}

for an extended period of time can damage the green stems and leaves [3]. During the reproductive stage, the optimum temperatures for seed maturation are 19$20^{\circ} \mathrm{C}$. At this stage, severe chilling stress reduces soybean yield mainly by reducing seed size and delaying maturity [4-6]. Therefore, unravelling the mechanism of how plants avoid low temperature damage could provide valuable information for breeding and improving soybean yield in agricultural production.

Plants have evolved sophisticated mechanisms to cope with ever-changing environments. After a period of exposure to low temperatures (nonfreezing temperatures), plants exhibit greater tolerance to freezing temperature, which is called cold acclimation [7, 8]. Cold acclimation comprises comprehensive physiological, biochemical and molecular actions [9]. At the physiological level, plenty of protective substances including proline, soluble sugars and cold-resistant proteins, etc. are synthesized [10]. At 
the molecular level, the cold signal is first perceived by cell membrane fluidity, ion channels (i.e., $\mathrm{Ca}^{2+}$ channels) and electrophysiology, which then induces plasma membrane rigidification and activates the $\mathrm{Ca}^{2+}$ channel, leading to the influx of $\mathrm{Ca}^{2+}$ into the cytosol. In Arabidopsis, cold signals activate a receptor-like cytoplasmic kinase cold-responsive protein kinase 1 (CRPK1), in which CRPK1 phosphorylates and promotes the accumulation of 14-3-3 proteins in the nucleus. 14-3-3 proteins involve in many physiological processes in plants, including responses to abiotic stress [11]. In the nucleus, phosphorylated 14-3-3 proteins promote the degradation of CBFs via the $26 \mathrm{~S}$ proteasome [12].

CBFs function as key transcription factors in the nucleus and regulate a complex cold signal transduction network [13]. There are three cold-induced $C B F$ genes, CBF1-3 (CBF1/DREB1B, CBF2/DREB1C and CBF3/ $D R E B 1 A)$, arranged in tandem along a chromosome in Arabidopsis. The expression of $C B F s$ is tightly regulated by several upstream regulators [14]. ICE1 (Inducer of CBF Expression 1), which belongs to basic helix-loophelix (bHLH) family protein, directly binds to $C B F 3$ promoter and activates the expression of $C B F 3$ upon cold stress [15]. The mutation of ICE1 blocks CBF3 level and decreases freezing tolerance [15-17]. CBFs regulate 10$20 \%$ of downstream COLD REGULATED (COR) genes in Arabidopsis in responsive to cold treatment [18-20]. COR genes contain a dehydration-responsive element/Crepeat (DRE/CRT) cis-element as common features in their promoter region. CBFs bind to the DRE/CRT ciselements and activate $C O R$ genes, which confer chilling and freezing tolerance to plants [21-24]. COR15a is the most distinctive COR protein that localizes at the membrane and maintains the integrity of cell membranes under cold stress [25]. In addition to the CBF-dependent pathway in Arabidopsis, COR gene is also modulated by several CBF-independent regulators. For example, BRASSINAZOLE RESISTANT1 (BZR1), a transcription factor in the brassinosteroid (BR) signal transduction pathway, modulates plant tolerance to low temperature through other COR genes (PYR1-LIKE 6 (PYL6) and SUPPRESSOR OF OVEREXPRESSION OF CO1 (SOC1) uncoupled with CBFs [26]; HOS9, encodes a homeodomain transcription factor, modulates cold signaling through a CBF-independent pathway in Arabidopsis [27]. Thus, plant's response to low temperature is a complex process in Arabidopsis.

In soybean, great efforts have been made to understand and improve plant cold tolerance. Proteomic analyses have also revealed that cold-tolerant soybean produces more protective substances [3]. Transcriptome analyses have identified many cold-responsive genes in soybean, including $C B F$ genes [28, 29]. Indeed, the ectopic expression of soybean $C B F s$ enhanced the freezing tolerance by elevating the levels of AtCOR47 and AtRD29a transcripts in Arabidopsis [30]. The GmDREB3 gene, a member of the DREB A-5 subfamily, has been specifically induced by cold stress, with overexpression of GmDREB3 in Arabidopsis enhancing plant cold tolerance [30,31]. Several soybean COR genes have also been identified as positive regulators of plant cold tolerance [32-35]. For example, Soybean SCOF-1, MYB, WRKY, $b Z I P$ and Zinc Finger-type transcription factor genes have been shown to mediate plant cold tolerance [3235]. These preliminary results indicate that soybean has multiple mechanisms to cope with low temperature stress. However, the detailed mechanisms of soybean tolerance to low temperature stress remain largely unknown.

Previously, we identified AtTCF1 (Tolerant to Chilling and Freezing 1) as a COR gene in Arabidopsis. AtTCF1 encodes an RCC1-like protein and responds specifically to cold stress by transcription and protein accumulation in Arabidopsis [36]. Loss of AtTCF1 leads to reduced $B C B$ (blue copper-binding) level and lignin content, results in cold tolerance. The results have revealed the important role of AtTCF1-mediated cell wall remodeling under cold stress. In this study, we identified a putative ortholog of AtTCF1 in soybean, GmTCF1a, which is specifically regulated in response to cold stress. Intriguingly, ectopic expression of GmTCF1a in Arabidopsis resulted in elevated levels of COR15a and enhanced plant tolerance to freezing stress. These results indicate that GmTCF1a positively regulates cold tolerance in soybean.

\section{Results}

\section{Identification of GmTCF1s in soybean}

To identify homologs of Arabidopsis TCF1 (AtTCF1) in soybean, we retrieved protein sequences containing RCC1 domains from Arabidopsis and soybean using a HMM (Hidden Markov Model) search (PF00415 and PF13540), and 55 non-redundant soybean genes and 25 non-redundant Arabidopsis genes were confirmed as homologs of AtTCF1. A phylogenetic tree was constructed to examine the classification and evolutionary history of these RCC1-like proteins (Fig. 1). All of these RCC1 like proteins were clearly divided into 9 major clades. AtTCF1 and four proteins (Glyma.02G250700, Glyma.14G066000, Glyma.11G223000 and Glyma.18G034600) were grouped in clade V (Fig. 1). This result indicates that AtTCF1 and these four proteins are more closely related than others. Then Glyma.02G250700 was named as GmTCF1a, Glyma.14G066000 as GmTCF1b, Glyma.11G223000 as GmTCF1c and Glyma.18G034600 as GmTCF1d based on the evolutionary relationship with AtTCF1. 


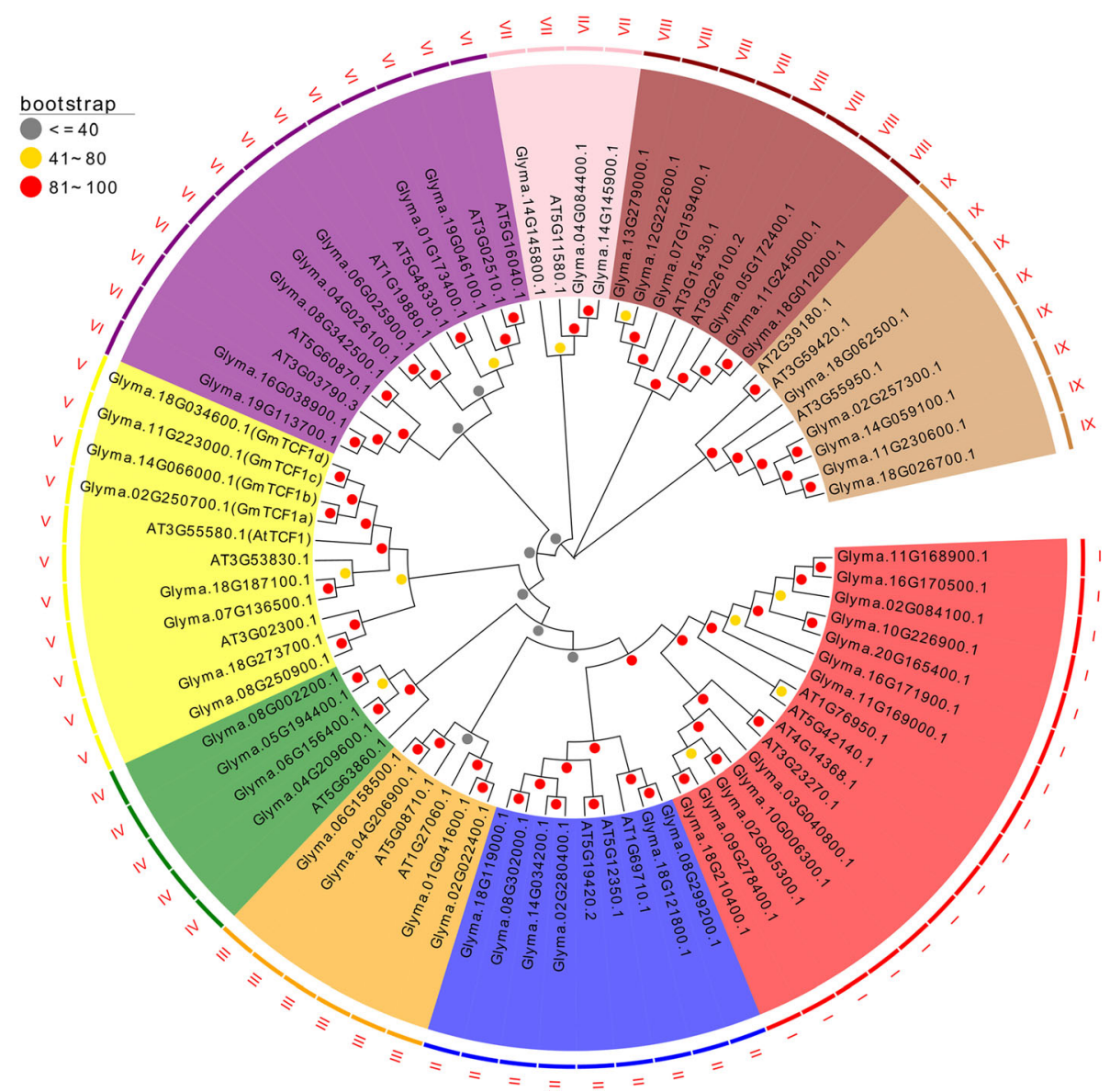

Fig. 1 Phylogenetic analysis of RCC1-like proteins from Glycine max $(\mathrm{Gm})$ and A. thaliana (At). Clustal W was used to align 80 RCC1-like proteins, and MEGA X software was employed to construct a neighbor-joining phylogenetic tree with 1000 bootstrap replications. RCC1-like proteins were separated into nine clades from I to IX. Different clades were distinguished by different colors

Furthermore, we investigated the synteny of TCF1 homologous genes between the soybean and Arabidopsis genomes. By searching the plant genome duplication database (PGDD), we found that AtTCF1 displayed collinearity with four GmTCF1s genes (GmTCF1a, GmTCF1b, GmTCF1b and GmTCF1d) in soybean (Supplementary Fig. S1). Next, we performed a synteny analysis with the AtTCF1 coding sequence using McScanX in leguminous plants. The same four GmTCF1s were found in soybean (Fig. 2a). However, only one collinear gene of AtTCF1 was found in Medicago and Lotus, respectively (Fig. 2a). These collinearity results suggest that AtTCF1 and the four GmTCF1s genes may be traced to a common ancestor, and twice the whole genome duplication produced more copies of AtTCF1 homologous genes in soybean. To explore the evolutionary relationship of TCF1 homologous genes in different species, a phylogenetic tree with 21 TCF1 homologs from 13 species was constructed. As shown in Fig. 2b, the 21 TCF1 homologs can be divided into 3 clades. Clade I contains one Arabidopsis and four legume dicot species, Clade II species belong to non-legume dicotyledonous plants, and the remaining TCF1 homologs from monocots (Zea mays, Oryza sativa, B. distachyon and Sorghum bicolor) were grouped in clade-III (Fig. 2b). AtTCF1 was grouped with GmTCF1a and GmTCF1b in the clade I, indicating that AtTCF1 has remarkably high homology with genes in legume plants and low homology with monocot genes (Fig. 2b). Taken together, GmTCF1a and GmTCF1b are more closely related to AtTCF1 than to monocots, which may be partly due to the fact that both soybean and Arabidopsis are dicotyledonous plants.

\section{Considering GmTCF1a as a putative orthologous gene of AtTCF1}

To explore the organ-specific expression pattern of these GmTCF1s, we retrieved the RNA sequencing expression data of TCF1 homologs from public data (http://bar. utoronto.ca/) and arranged for heatmap production. As shown in Fig. 3a, AtTCF1 was strongly expressed, while GmTCF1s were lowly expressed in flowers. The GmTCF1a gene was mainly expressed in the aerial parts 


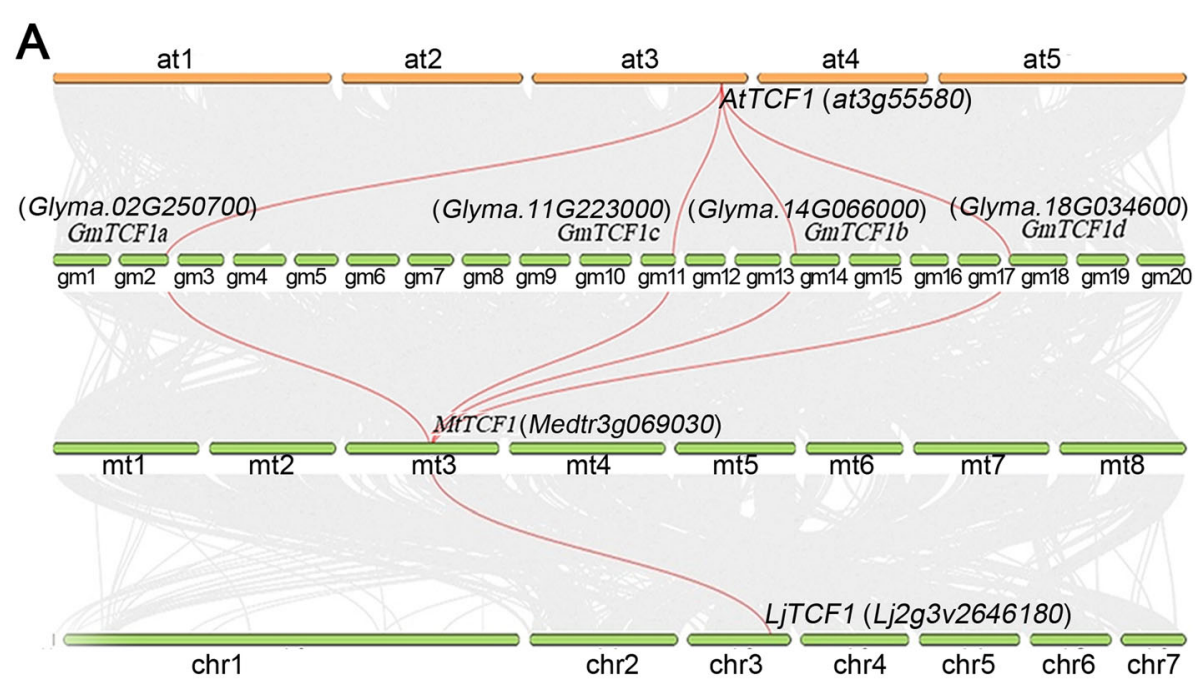

B

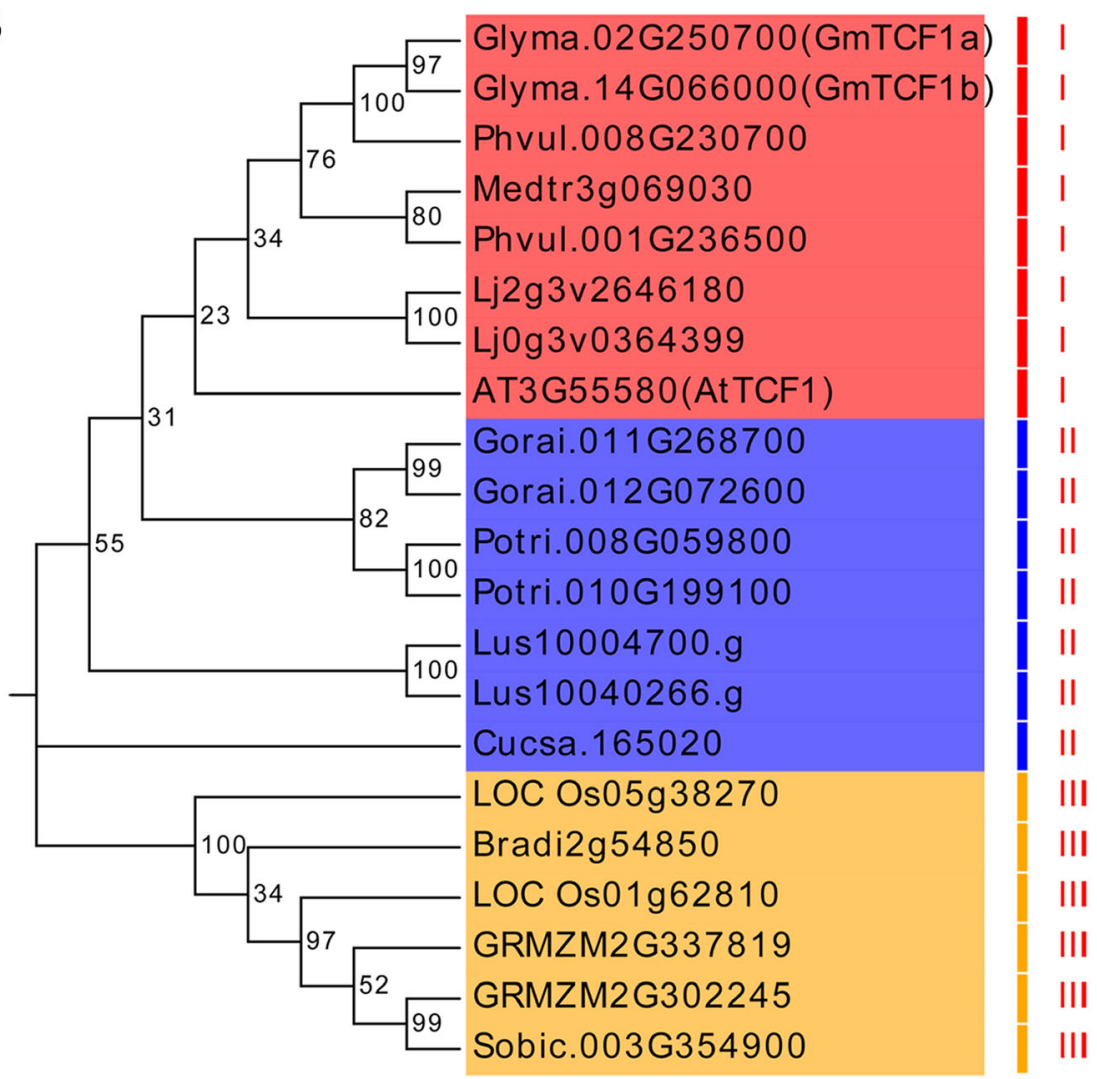

Fig. 2 The synteny and phylogenetic analysis of TCF1 with its homologs. A MCScanX was used for synteny analysis of AtTCF1 with homologous genes in Glycine max, Medicago truncatula and Lotus japonicus. Gray lines represent collinear blocks within species, while red lines represent syntenic TCF1 gene pairs. B Phylogenetic tree of GmTCF1s and their homologs from various organisms, including A. thaliana, Oryza sativa, Glycine Max, Medicago truncatula, Lotus japonicus, Phaseolus vulgaris, Populus trichocarpa, Linum usitatissimum, Gossypium raimondii, Zea mays, $B$. distachyon, Sorghum bicolor and C. sativus. Bootstrap support values of 1000 replicates are given at each node. RCC1-like proteins were separated into three clades from I to III

of the plant (leaves, shoot apical meristem and pods) and had lower expression in flowers, nodules and roots. The expression of the remaining homologs (GmTCF1b, GmTCF1c and GmTCF1d) was also high in nodules compared to GmTCF1a (Fig. 3a), suggesting that GmTCF1b, GmTCF1c and GmTCF1d may acquire new function(s) in the underground parts of soybean. Further correlation analysis found that AtTCF1 had a low 


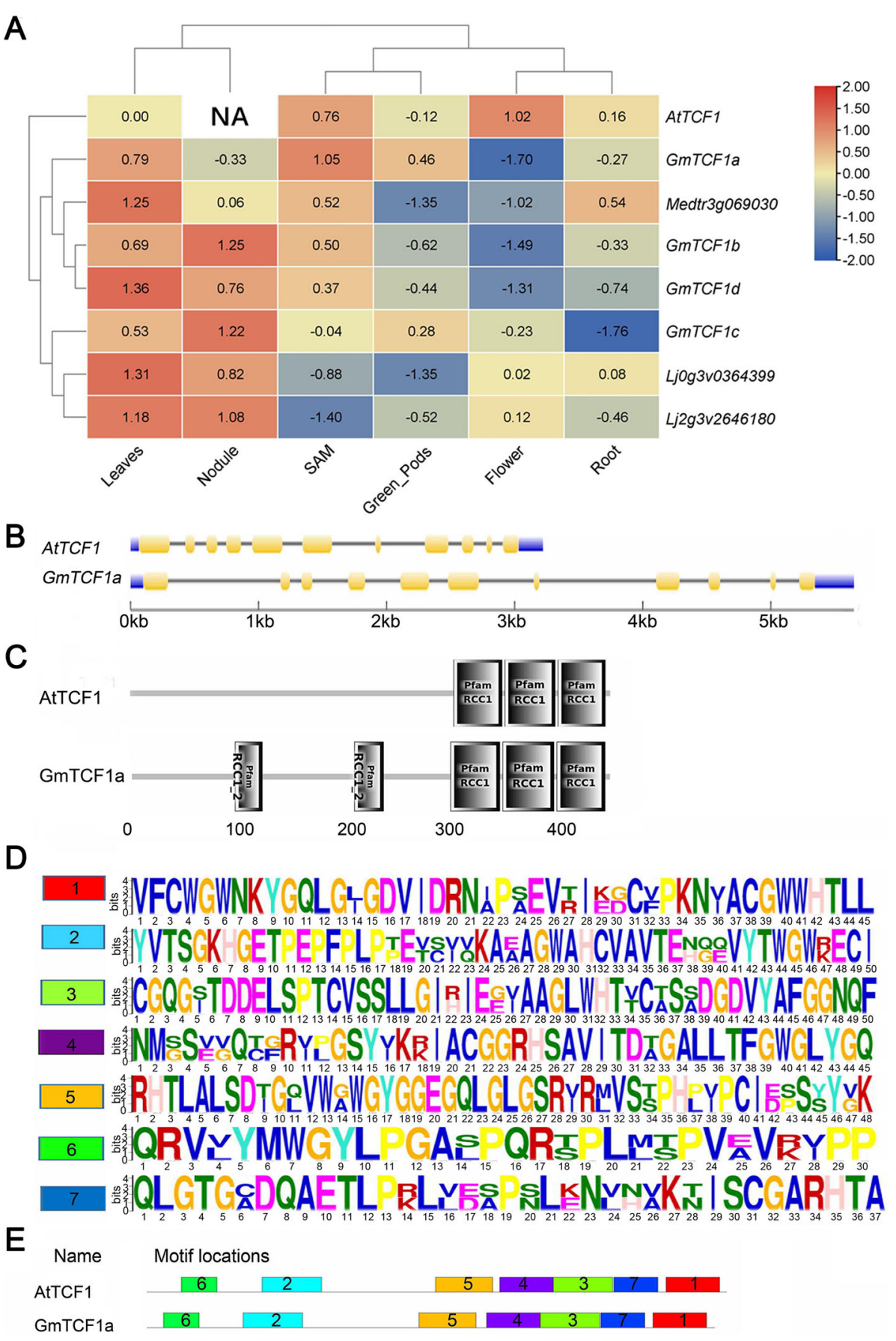

Fig. 3 Expression pattern, domain and motifs analysis of AtTCF1 homologs. A Expression profiles of AtTCF1 homologs from leguminous plants. SAM, shoot apical meristem; NA, no data for AtTCF1 in nodule. B The intron-exon structure of GmTCF1a and AtTCF1 genes. Introns and exons are represented by gray lines and yellow boxes, respectively. C Domain analysis of GmTCF1a and AtTCF1. The gray boxes show the RCC1 domain. D Conserved motifs of GmTCF1a and AtTCF1. Both GmTCF1 and AtTCF1 contain seven motifs indicated by different colors. E Distribution of conserved motifs along with the protein sequence. The motifs, numbered 1-7, are shown in different colored boxes. GmTCF1 and AtTCF1 share all the seven motifs

correlation with the expression pattern of GmTCF1a, while AtTCF1 had a strong negative correlation with the rest genes except GmTCF1a (Fig. S2). From the phylogenetic tree, GmTCF1a and GmTCF1b are the most homologous genes of AtTCF1 (Fig. 2b). However, we found a low temperature-related cis-acting element [37] existing in the GmTCF1a promoter, but not in the GmTCF1b promoter (Fig. S3). Thus, GmTCF1a is 
considered as a putative orthologous gene of AtTCF1 for further study.

The GmTCF1a gene has a gene structure similar to that of AtTCF1. Both genes contain 11 exons and 10 introns, but the GmTCF1a gene is much larger than AtTCF1 because most of the introns in GmTCF1a are much longer than those in AtTCF1 (Fig. 3b). GmTCF1a encodes a predicted protein of 477 amino acids with a calculated MW (molecular weight) of $50.199 \mathrm{kDa}$ and a PI (protein isoelectric point) of 6.01. Interestingly, GmTCF1a contains 5 RCC1-like domains, two more than AtTCF1 (Fig. $3 \mathrm{c})$, and shares $65 \%$ identity with AtTCF1 in its amino acid sequence (Fig. S4). The protein sequences of GmTCF1a and AtTCF1 were then subjected to MEME online analysis to find the common motifs. Seven conserved motifs were found in GmTCF1a and AtTCF1, which are consistent with the distribution of the motifs along with protein sequences (Fig. 3d and e). The conserved motifs indicate that GmTCF1a and AtTCF1 might exert functionally identical effects.

\section{Specific response of GmTCF1a gene to cold}

The expression of GmTCF1a gene in response to common adverse environmental conditions, such as cold, high soil salinity, drought and ABA was investigated by quantitative real-time PCR (qRT-PCR) [36]. Total RNA was extracted from soybean leaves treated with $4{ }^{\circ} \mathrm{C}$, $200 \mathrm{mM} \mathrm{NaCl}, 15 \%$ PEG and $100 \mu \mathrm{M} \mathrm{ABA}$ at different time points. As shown in Figs. 4a and S5a, GmTCF1a had a very low expression level under normal conditions. Upon cold treatment, GmTCF1a expression was dramatically induced at $6 \mathrm{~h}$ and reached the highest level at 12 $\mathrm{h}$ (Figs. 4a and S5a). However, the expression of GmTCF1a did not change under $\mathrm{NaCl}, \mathrm{ABA}$ and PEG treatments (Figs. 4b, c, d and S5b, c, d). In addition, the organ-specific expression pattern of GmTCF1a was examined using total RNA isolated from leaves, stems, roots and nodules of soybean plants with and without cold treatment. GmTCF1a was expressed at low levels in all organs tested under normal conditions; in sharp contrast, GmTCF1a was induced in these organs by cold treatment with the exception of the nodule and showed the highest expression level in the leaf (Figs. 4e and S5e).

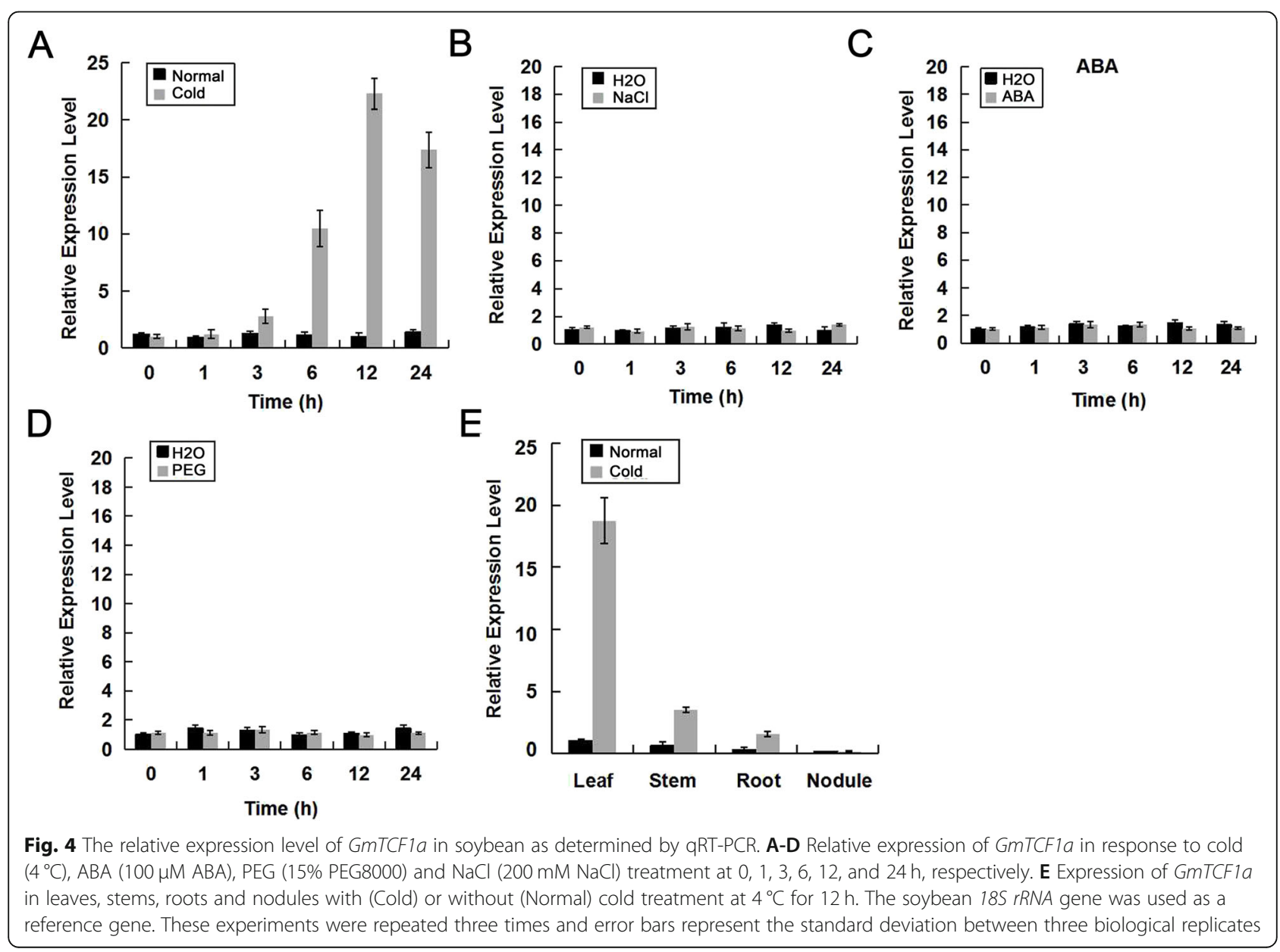


Taken together, these results suggest that GmTCF1a is a cold-responsive gene that may mediate plant responses to cold stress.

\section{Preferential expression of GmTCF1a in vascular tissues}

To further examine the cell/tissue-specific expression of GmTCF1a, we constructed a binary vector containing a $2.0 \mathrm{~kb}$ GmTCF1a promoter that drives the $\beta$-glucuronidase (GUS) gene and then transformed GmTCF1apro: GUS into soybean root hairs and Arabidopsis. GUS histochemical staining revealed that GmTCF1a was expressed mainly in the root stele of soybeantransformed hairy roots, and its expression became much higher after cold treatment (Fig. 5a). In Arabidopsis plants expressing GmTCF1apro:GUS, GUS staining was detected only in the veins of cotyledons under normal conditions, whereas very strong GUS staining was observed in the entire cotyledons and hypocotyls after exposure to cold stress (Fig. 5b). These results indicate that GmTCF1a is highly induced by cold in both shoots and roots.

\section{GmTCF1a as a nuclear protein}

To examine the subcellular localization, we made a construct harboring a GFP-GmTCF1a fusion gene under the control of the CaMV $35 \mathrm{~S}$ promoter and generated transgenic Arabidopsis plants. Seven-day-old transgenic Arabidopsis seedlings were treated at $22^{\circ} \mathrm{C}$ and $4{ }^{\circ} \mathrm{C}$ for 12 $\mathrm{h}$, respectively. Confocal microscopy revealed that the GFP-GmTCF1a fusion proteins were localized in the
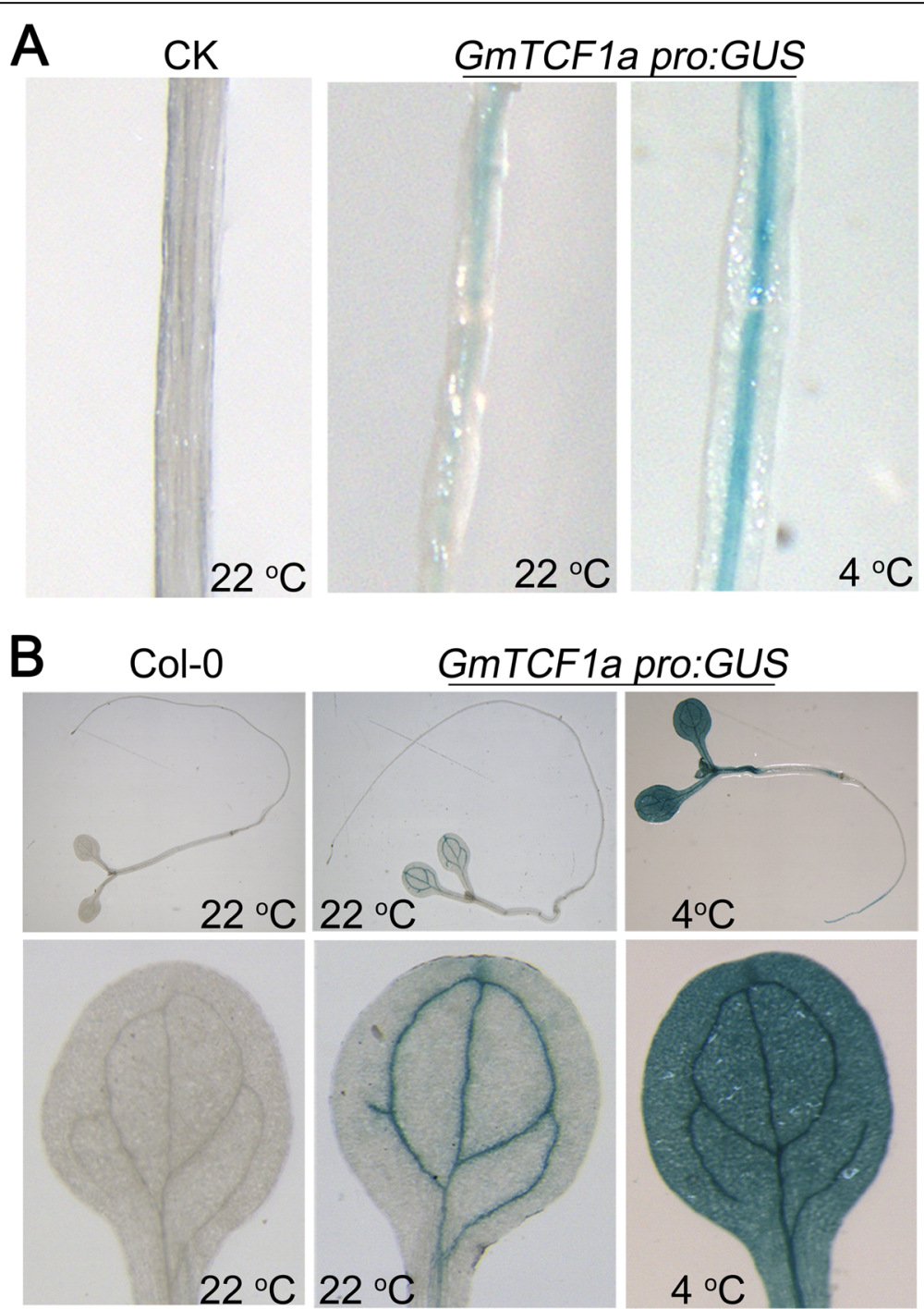

Fig. 5 Histochemical staining of transgenic plants expressing GmTCF1apro:GUS. A GUS staining of soybean hairy roots expressing GmTCF1apro:GUS fusion with and without cold treatment. CK, Soybean hairy roots without GmTCF1apro:GUS fusion; B GUS staining of transgenic Arabidopsis seedlings containing GmTCF1apro:GUS fusion with and without cold treatment. Wild-type Arabidopsis Col-0 is defined as a control 
nucleus under normal condition, contrasting sharply with the ubiquitous localization of GFP in the entire root cell (Fig. 6). Cold stress did not alter nuclear localization of GmTCF1a (Fig. 6). Thus, GmTCF1a and AtTCF1 display the same subcellular localization, and play roles in the nucleus during cold stress [36].

\section{Enhancement of freezing tolerance in Arabidopsis plants by ectopic expression of GmTCF1a}

To investigate whether GmTCF1a acts as a key regulator of the response of plants to cold stress, we constructed a binary vector harboring 35S:GmTCF1a and transformed it into Arabidopsis plants. After three generations of selection, we obtained two homozygous transgenic lines that constitutively overexpress GmTCF1a (Fig. 7a). Three-week-old transgenic plants and wild-type plants were treated at $4{ }^{\circ} \mathrm{C}$ for 7 days before being subjected to $-8^{\circ} \mathrm{C}$ for $2.5 \mathrm{~h}$. Survival rates were calculated after 7 days of recovery under normal growth conditions. The survival rates of the two representative 35S:GmTCF1a transgenic Arabidopsis lines from independent transgenic events were about 47\% (line \#14-4) and 42\% (line $\# 17-3)$, respectively; by contrast, the survival rate of wild-type plants was only 15\% (Fig. $7 \mathrm{~b}$ and c). The result indicates that overexpression of GmTCF1a greatly elevates the tolerance of the transgenic plants to low temperature.
Cold stress can destroy the plant cell walls and cause the cytosol to leak out. Therefore, the lower rate of electrolyte leakage reflects higher cold tolerance. An electrolyte leakage assay was performed to compare the freezing tolerance between GmTCF1a overexpression plants (\#144 and \#17-3) and wild-type plants. As shown in Fig. 7d, there were no significant difference in electrolyte leakage rates between transgenic plants and wild-type plants when treated at $25,0,-2$ and $-4{ }^{\circ} \mathrm{C}$ for $0.5 \mathrm{~h}$; however, the percentages of electrolyte leakage were significantly lower in GmTCF1a overexpression plants than in wild-type plants when the temperature was reduced to -6 and $-8^{\circ} \mathrm{C}$ (Fig. $7 d)$. Although the electrolyte leakage of $\# 17-3$ were slightly higher than \#14-4, but no significant differences were found. Taken together, the result indicates that the overexpression of GmTCF1a can improve the freezing tolerance of Arabidopsis plants.

We also tested the role of overexpression AtTCF1 in Arabidopsis. As shown in Fig. S6, the survival rates of two independent homozygous 35S:AtTCF1 transgenic Arabidopsis lines were about 37\% (line \#3-6) and 48\% (line \#12-2), respectively; by contrast, the survival rate of wild-type plants was only $19 \%$. The result indicates that overexpression of AtTCF1 greatly elevates the tolerance of the transgenic plants to low temperature. The electrolyte leakage assay also demonstrated that AtTCF1 overexpression plants reduced the percentage of leakage at $-8{ }^{\circ} \mathrm{C}$ (Fig. S6d). In summary, these results indicate

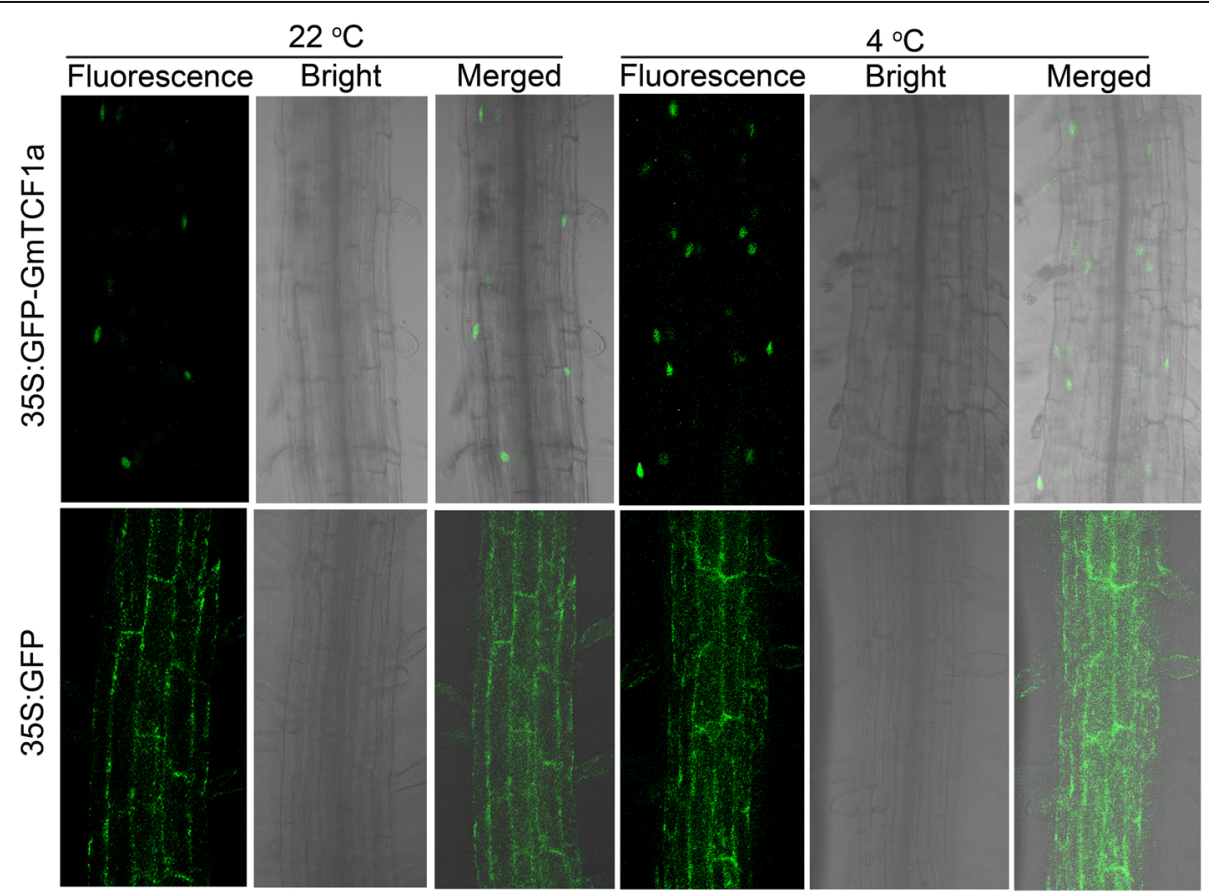

Fig. 6 GmTCF1a localizes in the nucleus. Seven-day-old transgenic Arabidopsis seedlings expressing GFP-GmTCF1a and GFP were treated at $22^{\circ} \mathrm{C}$ and $4{ }^{\circ} \mathrm{C}$ for $12 \mathrm{~h}$ before imaging analysis. The root cells in the elongation zone were observed using a $488 \mathrm{~nm}$ laser confocal microscope. Fluorescence, GFP channel; Bright, bright field; Merged, combination of GFP channel and bright field channel 
A

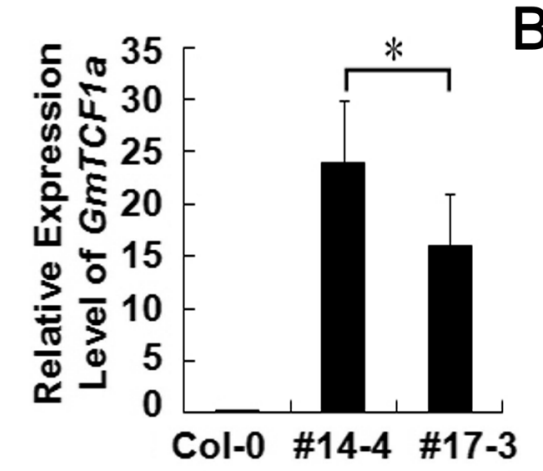

C

$-8{ }^{\circ} \mathrm{C}$

$(2.5 \mathrm{~h})$

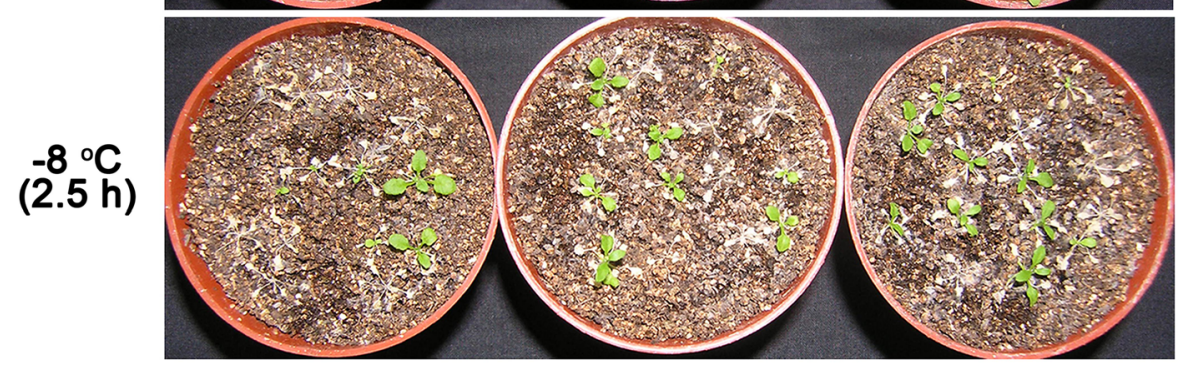

D
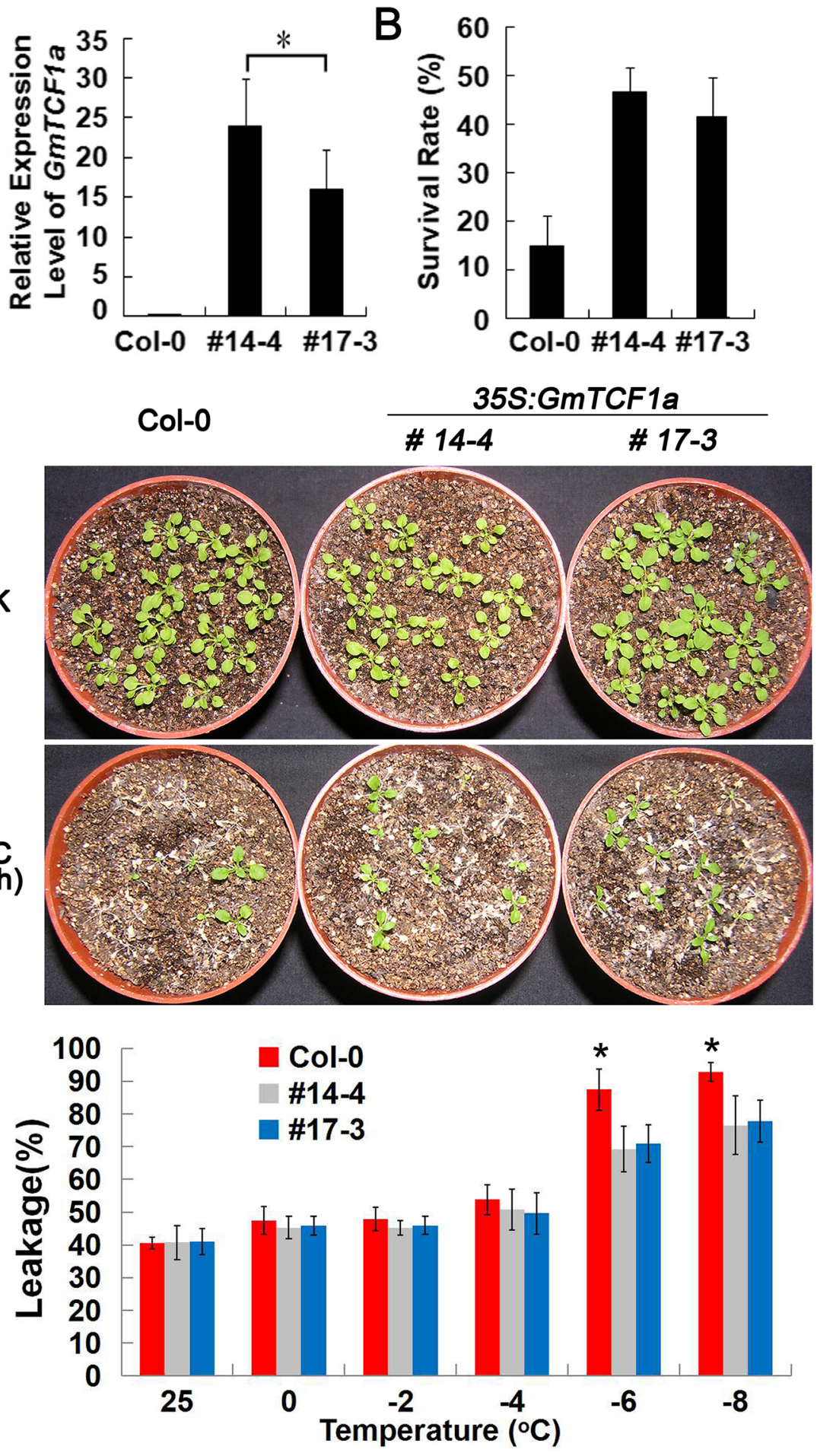

Col-0

35S:GmTCF1a

\section{\# 14-4 \#17-3}

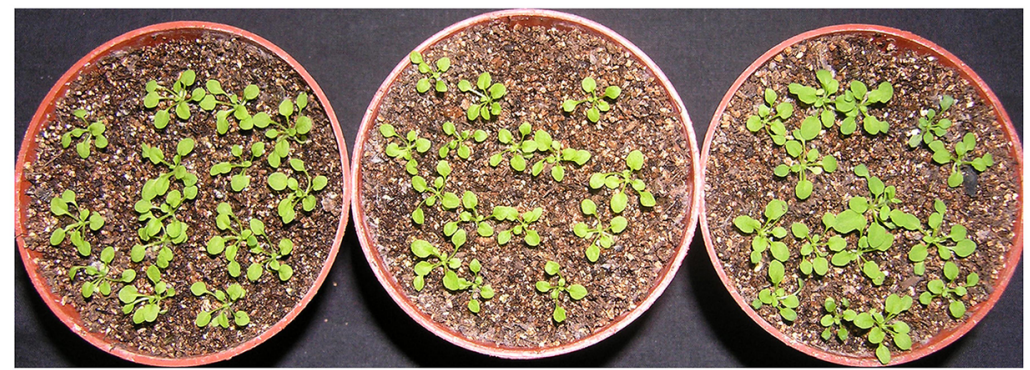

Fig. 7 Increase in cold tolerance by overexpression of GmTCF1a in Arabidopsis. A The expression level of GmTCF1a in two 35S:GmTCF1a transgenic plants. The UBC gene was used as a control. B The survival rate of wild-type and 355:GmTCF1a lines after freezing treatment from b. $\mathbf{C}$ Freezing assay of wild-type and two transgenic Arabidopsis lines (\#14-4 and \#17-3) at $-8^{\circ} \mathrm{C}$ for $2.5 \mathrm{~h}$ after cold acclimation. Photographs were taken 7 days after freezing treatment. D Electrolyte leakage from wild-type and two 35S:GmTCF1a lines after being treated at temperatures of 0, $2,-4,-6$, and $-8^{\circ} \mathrm{C}$ for $0.5 \mathrm{~h}$. Error bars represent the Mean \pm SD from three technical replicates. One-way ANOVA with a Tukey test was performed to explore the significant differences at -6 and $-8^{\circ} \mathrm{C}$ treatment, and ${ }^{*}$ indicates $P<0.05$. These experiments were repeated three times and obtained the same results 
that overexpression of AtTCF1 or GmTCF1a can improve the freezing tolerance of Arabidopsis plants.

\section{Upregulation of COR15a by GmTCF1a overexpression in Arabidopsis plants}

To determine whether overexpression of GmTCF1a affects cold-responsive gene expression in Arabidopsis, total RNA was extracted from cold-treated transgenic and wild-type Arabidopsis seedlings. Due to the same freezing tolerance phenotype of line \#17-3 and \#14-2, we selected line $\# 17-3$ as a representative transgenic plants for gene expression analysis. Six cold responsive genes CBF1, CBF2, CBF3, COR15a, COR47 and RD29a were selected $[21,24,30]$ and examined by qRT-PCR. As a result, the expression of $C B F 1, C B F 2, C B F 3$, $C O R 47$ and $R D 29 a$ was not altered in the line \#17-3 compared to wild-type plants. However, the expression of COR15a was much higher in transgenic plants than in wild-type plants at the tested time points (Fig. 8). This result suggests that GmTCF1a enhances the cold tolerance of plants by up-regulating COR15a expression. We also investigated the level of COR15a in AtTCF1 overexpression transgenic lines (\#12-2), and COR15a was also higher in transgenic plants than in wild-type plants (Fig. S7). In summary, the increased cold tolerance in overexpressed AtTCF1 or GmTCF1a plants is associated with the up-regulated levels of COR15a.

\section{Discussion}

Low temperature is one of the primary abiotic stresses, which negatively affects the growth and productivity of soybean. Identifying the determinants of soybean tolerance to low temperature is crucial for the genetic improvement of soybean stress tolerance. In this study, we identified GmTCF1a in soybean as a putative orthologous gene of Arabidopsis TCF1, which is specifically induced by cold stress and regulates freezing tolerance in Arabidopsis. These findings reveal a conserved mechanism by which plants respond to low freezing temperatures and provide novel insights into the genetic improvement of freezing tolerance in soybean.

\section{GmTCF1a as a putative orthologous gene of AtTCF1}

Several RCC1 family proteins, such as UVR8, TCF1, RUG3 and SAB1, have been characterized and exhibit different biological functions in response to abiotic stresses [36, 38-43]. In Arabidopsis, AtTCF1 regulates plant tolerance to freezing stress by reprogramming cell wall properties [36]. Like Arabidopsis, soybean is also a temperate plant and is susceptible to low temperatures. We hypothesized that soybean might adopt a similar mechanism to cope with low temperature stress. Indeed, we found four homologous genes of AtTCF1 in soybean. Apparently, GmTCF1s exhibit evolutionary diversity in soybean. GmTCF1s contain several RCC1-like domains (Fig. S8) and share a high degree of sequence identity and similarity (Fig. S4). It is well known that wholegenome duplication (WGD) plays a central role in the expansion of the soybean gene family [44], with two independent duplications occurring approximately 59 and 13 million years ago, resulting in $75 \%$ of genes in multiple copies in soybean [45]. Therefore, four GmTCF1s can be evolved through WGD. Among the four GmTCF1s, GmTCF1a is considered a putative orthologous gene of AtTCF1. Firstly, phylogenetic analysis showed that GmTCF1a was the closest homolog of AtTCF1 (Fig. 2b). Secondly, a low-temperature-related cis-acting element was present in the GmTCF1a promoter (Fig. S3). Finally, GmTCF1a was barely expressed in legume-specific organ-nodules (Fig. 3a). Thus, the GmTCF1a gene has a high degree of evolutional conservation in soybean. In addition, GmTCF1a has longer introns than AtTCF1 in their genomic sequence, and three putative splicing variants of GmTCF1a from the phytozome database were found, whether these variants are responsive to cold stress or cold tolerance need to be further investigated.

\section{GmTCF1a as a functional ortholog of AtTCF1}

Indeed, our further experiments demonstrated that GmTCF1a is a functional ortholog of TCF1. First, GmTCF1a and AtTCF1 are specifically responsive to cold temperature [36]. Under normal conditions, both GmTCF1a and AtTCF1 were expressed at very low levels in leaves, stems and roots. After $12 \mathrm{~h}$ of cold stress, the expression of GmTCF1a and AtTCF1 was strongly induced in leaves, stems and roots (Figs. 4e and 5) [36]. Most importantly, the expression of both genes was specifically and highly induced by cold stress, but not by ABA, PEG and salt stress (Fig. 4a,b,c,d) [36]. In transgenic soybean roots, despite the fact that a $6 \mathrm{~h}$ GUS response at $37^{\circ} \mathrm{C}$ might reverse the cold-induced upregulation of GmTCF1apro:GUS expression in certain cells, GmTCF1a was highly induced in vascular tissue by cold stress, while GmTCF1a transcription was predominantly induced on the aboveground of Arabidopsis (Fig. 5). Second, GmTCF1a and AtTCF1 display the same subcellular localization, and they are both localized in the nucleus (Fig. 6). Third, both GmTCF1a and AtTCF1 mediate the response of Arabidopsis to freezing temperature. We showed that ecotopic GmTCF1a or AtTCF1 enhanced plant tolerance to freezing temperatures (Figs. 7 and S6). Finally, like AtTCF1, GmTCF1a involved in freezing tolerance is independent of CBFs [36], because ecotopic expression of GmTCF1a does not alter the expression of $C B F s$ in Arabidopsis. These results demonstrate that GmTCF1a is indeed a functional ortholog of AtTCF1 and that soybean may also have a 


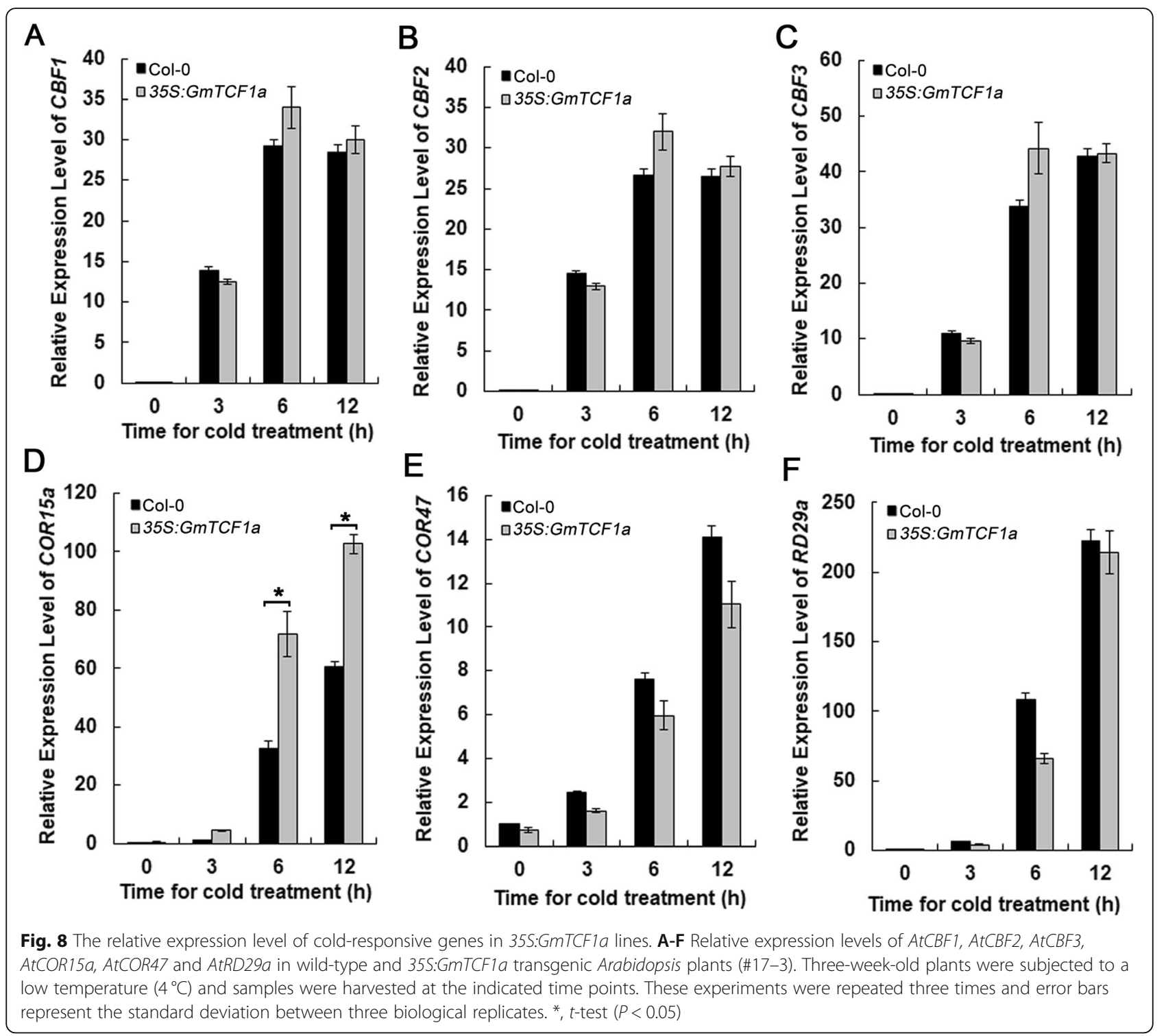

conserved TCF1-mediated mechanism in response to freezing and cold.

\section{GmTCF1a as a positive regulator of freezing tolerance} In Arabidopsis, we showed that the loss of AtTCF1 function exhibited enhanced tolerance to freezing temperatures [36]. In this study, we showed that overexpression of AtTCF1 also dramatically enhanced freezing tolerance in transgenic Arabidopsis (Fig. S6), suggesting a complex mechanism for AtTCF1 in the cold signaling pathway. However, we found that constitutive expression of either GmTCF1a or AtTCF1 elevated the level of COR15a. COR15a encodes a chloroplast-targeting polypeptide, and constitutive expression of COR15a enhances its in vivo freezing tolerance to chloroplasts under cold acclimation or nonacclimated treatment in Arabidopsis $[46,47]$. Thus, it is possible that enhancing freezing tolerance of chloroplasts may lead to freezing tolerance in transgenic plants. The manner in which GmTCF1a regulates COR15a levels remains unclear. RCC1-like protein usually associates with downstream genes and regulates their expression by affecting histone modifications in the gene promoter region [36, 41, 48, 49], and we speculate that GmTCF1a may influence histone modifications in the COR15a promoter or that GmTCF1a interacts with an unknown gene protein, which in turn regulates COR15a levels. In addition, it is known that strict control of lignin homeostasis in the cell wall is required for freezing tolerance [50]. Reduced lignin deposition within the cell wall in the tcf1 mutant during cold stress may increase cell wall permeability and protect the cells from freezing damage [36]. Overexpression of AtTCF1 is likely to also enhance plant stress tolerance and maintain the lignin content at an optimal 
level. We speculate that overexpression of GmTCF1a has a significant effect on influencing cellular lignin content. Further studies on lignin dynamics in response to freezing temperature and the role of GmTCF1a in this process will provide novel insights into the mechanisms of freezing tolerance in plants.

\section{Conclusions}

In conclusion, this study reports a RCC1-like gene, GmTCF1a, which is a novel gene associated with cold acclimation in soybean. GmTCF1a is specifically induced by cold stress and enhances freezing tolerance to transgenic Arabidopsis. It is still necessary to investigate the exact molecular and physiological mechanisms by which GmTCF1a and its paralogs regulate freezing tolerance in soybean. The application of GmTCF1a in improving cold tolerance in soybean is also worth exploring.

\section{Methods}

\section{Identification of GmTCF1s and structure analysis}

The coding sequence (CDS) of AtTCF1 was retrieved from the TAIR website (https://www.arabidopsis.org/). To identify soybean RCC1-like proteins, the soybean and A. thaliana protein sequences and genome annotations were downloaded from the Phytozome database (https:// phytozome.jgi.doe.gov/pz/portal.html). First, the HMM (Hidden Markov Model) of the RCC1 (PF00415 and PF13540) domain was obtained from the PFAM database (http://pfam.xfam.org/), and the soybean RCC1s (GmRCC1s) and Arabidopsis RCC1s (AtRCC1s) was predicted using the HMMER software. Second, the protein sequences were then used to construct HMMs [51]. Third, the proteins containing conserved RCC1 domains were considered as GmRCC1s and AtRCC1s. The RCC1 domains were verified by SMART software (http:// smart.embl-heidelberg.de/) and the PFAM database. Finally, using AtRCC1s sequence as a query to search the soybean proteome (BLASTP), proteins that met the BLASTP threshold conditions (E-value $\leq 1 \mathrm{e}-5$ and identity $250 \%$ ) were retained as the final $\mathrm{GmRCC} 1 \mathrm{~s}$ and AtRCC1s family members. PI and MW were analyzed by the Compute PI/MW tool (http://expasy.org/tools/pi_ tool.html). The gene structures of GmTCF1a and AtTCF1 were drawn based on cDNA sequences using the online software GSDS 2.0 (http://gsds.gao-lab.org/) [52]. The promoter sequence of GmTCF1s with a length of $2000 \mathrm{bp}$ upstream of the translation site (ATG) was obtained from Phytozome, and the cis-element was analyzed on the PlantCARE website (http://bioinformatics. psb.ugent.be/webtools/plantcare/html/). The RCC1 domains of GmTCF1a and AtTCF1 were predicted using SMART tools (http://smart.embl.de/smart/set_mode. cgi?NORMAL=1). The sequence alignment between GmTCF1a and AtTCF1 was performed using the
CLUSTALW program (https://www.genome.jp/toolsbin/clustalw) and MEGA 7.0 software [53].

\section{Phylogenetic and bioinformatics analysis}

The homologous protein sequences of GmTCF1a from A. thaliana, Glycine Max, Oryza sativa, Medicago truncatula, Phaseolus vulgaris, Populus trichocarpa, Linum usitatissimum, Gossypium raimondii, Zea mays, B. distachyon, Sorghum bicolor and C. sativus were retrieved from Phytozome using the BLASTP program, and the protein homologs of Lotus japonicus were retrieved from the Lotus base (https://lotus.au.dk/). The sequences were aligned by the CLUSTALW program and a phylogenetic tree was constructed using MEGA 7.0 software with the neighbor-joining method [53]. The collinearity analysis was performed by the MCScanX software (http://chibba. pgml.uga.edu/mcscan2/MCScanX.zip) and the figure was constructed by TBtools (https://github.com/CJChen/TBtools), respectively. The analysis of conserved motifs for GmTCF1a and AtTCF1 was performed by MEME online tool with default parameters except for a limit of seven numbers of motifs (http://meme-suite.org/ tools/meme) [54].

\section{Tissue expression pattern analysis}

Tissue-specific expression data (Normalized FPKM/ TMP) of eight AtTCF1 homologs were downloaded from eFP browser (http://bar.utoronto.ca/) and Lotus japonicus Expression Atlas (https://lotus.au.dk/expat/). Three biological replicates of these data from a subset of the tissues were used in the heatmap. Heatmap was built using row as scale type, the rows and columns of the heatmap was clustered. The correlation of the expressed data were analyzed using TBtools (SupCorrPlot function).

\section{Growth of soybean and treatments}

Soybean (G. $\max$ L.) Williams 82, a sequenced cultivar (originally from America), is a gift from the laboratory of Peter M. Gresshoff (University of Queensland, Australia). Williams 82 were grown on vermiculite under $16 / 8 \mathrm{~h}$ (light (6:00-22:00)/dark) photoperiod at $27^{\circ} \mathrm{C}$ for 3 weeks and then subjected to various abiotic stresses. For cold treatment, the pots were exposed to $4{ }^{\circ} \mathrm{C}$ at 9 : $00 \mathrm{am}$, and the first trifoliolate leaves were harvested after $1,3,6,12$, and $24 \mathrm{~h}$ treatments, respectively. For abscisic acid (ABA), PEG8000 and salt treatments, the roots of the seedlings were pulled out from the vermiculite and dipped into the solutions of $100 \mu \mathrm{M}$ ABA, 15\% PEG8000 and $200 \mathrm{mM} \mathrm{NaCl}$ at 9:00 am, respectively, and then the first trifoliolate leaves were harvested after $1,3,6,12$, and $24 \mathrm{~h}$ treatments.

To identify the expression pattern of GmTCF1a, soybean seeds were surface sterilized with $70 \%$ ethanol and 
then imbibed at $28^{\circ} \mathrm{C}$ for 3 days. The germinated seeds were then inoculated with $B$. japonicum USDA110 $(\mathrm{OD}=0.08)$ for $30 \mathrm{~min}$ and then transferred to the long test tubes with a low-nutrient solution containing $0.03 \mathrm{~g}$ $\mathrm{Ca}\left(\mathrm{NO}_{3}\right)-4 \mathrm{H}_{2} \mathrm{O}, 0.1 \mathrm{~g} \mathrm{CaCl}_{2}-2 \mathrm{H}_{2} \mathrm{O}, 0.1 \mathrm{~g} \mathrm{KH}_{2} \mathrm{PO}_{4}, 0.15 \mathrm{~g}$ $\mathrm{Na}_{2} \mathrm{HPO}_{4}-12 \mathrm{H}_{2} \mathrm{O}, 0.12 \mathrm{~g} \mathrm{MgSO}_{4}-7 \mathrm{H}_{2} \mathrm{O}, 0.05 \mathrm{~g} / \mathrm{L}$ ferric citrate, plus $1 \mathrm{~mL}$ of micro-element $\mathrm{H}_{2} \mathrm{BO}_{3} 2.86 \mathrm{mg}$, $\mathrm{MnSO}_{4} 1.81 \mathrm{mg}, \mathrm{ZnSO}_{4} 0.22 \mathrm{mg}, \mathrm{CuSO}_{4} 0.8 \mathrm{mg}$, and $\mathrm{H}_{2} \mathrm{MO}_{4} 0.02 \mathrm{mg} / \mathrm{L}$. The inoculated seedlings were grown at $27^{\circ} \mathrm{C}$ under a photoperiod of $16 / 8 \mathrm{~h}$ (light (6:00-22: $00) /$ dark) with a light intensity of $108.38 \mu \mathrm{mol} / \mathrm{m}^{2} \mathrm{~s}$. Whole plants were exposed to $4{ }^{\circ} \mathrm{C}$ at 9:00 am, then the first trifoliolate leaves, stems, roots and nodules were harvested at 21:00 pm, and samples were immediately frozen in liquid nitrogen and stored at $-80^{\circ} \mathrm{C}$ for RNA isolation.

\section{RNA isolation and real-time quantitative PCR (qRT-PCR)} Total RNA was extracted using the PureLink Plant RNA Reagent (ThermoFisher Scientific, cat. \#12322012). Firststrand cDNA was synthesized using the M-MLV reverse transcriptase enzyme (Promega). qRT-PCR was performed on an ABI PRISM 7500 real-time PCR system. The procedure for real-time PCR is as follows. The PCR reaction solution (total $20 \mu \mathrm{L}$ ) containing $10 \mu \mathrm{L}$ of SYBR Premix Ex Taq, $50 \mathrm{ng}$ cDNAs, $0.2 \mu \mathrm{M}$ of each primer, $0.4 \mu \mathrm{L}$ of ROX Reference DyeII. The PCR mixtures were heated at $95^{\circ} \mathrm{C}$ for $30 \mathrm{~s}$, followed by 40 cycles of amplification $\left(95^{\circ} \mathrm{C}\right.$ for $5 \mathrm{~s}, 60^{\circ} \mathrm{C}$ for $\left.34 \mathrm{~s}\right)$. The results were analyzed using 7500 system software with $2^{-\Delta \Delta C T}$ method. The $18 S$ rRNA gene was used as a control. The sequences of the qRT-PCR primers are shown in Table S1.

\section{Analysis of GmTCF1a pro:GUS expression pattern}

The fragment covering $2000 \mathrm{bp}$ immediately upstream of the GmTCF1a coding region was amplified with the primers listed in Table S1. The promoter was cloned into the PstI and BamHI sites preceding a GUS gene in the pCAMBIA1391 vector. The destination vector was transferred into A. tumefaciens strain GV3101. Floraldip method was used for stable transformation in Arabidopsis [55]. Transgenic plants were screened on MS medium containing $20 \mathrm{mg} / \mathrm{L}$ hygromycin B. After three generations of screening, we obtained two independent homozygous transgenic plants. The transformation of soybean hairy roots was performed based on a small amount of modification of soybean by Agrobacterium rizogenes-mediated methods [56]. GUS reactions of transgenic materials with and without cold treatment $\left(4{ }^{\circ} \mathrm{C}, 12 \mathrm{~h}\right)$ were performed for $6 \mathrm{~h}$ at $37^{\circ} \mathrm{C}$ in the dark. Chlorophyll was removed by washing several times with $70 \%$ ethanol and then representative images were photographed.

\section{GFP-GmTCF1a subcellular localization assay}

The coding sequence of GmTCF1a was amplified from cDNA with the primers shown in Table S1. The sequence was cloned into the $\mathrm{pEZR}(\mathrm{K})-\mathrm{LC}$ vector between the KpnI and BamHI sites after a GFP gene. This construct was then transformed into Col-0 (ordered from the Arabidopsis Biological Resource Center) via Agrobacterium-mediated transformation [55]. Homozygous transgenic plants were obtained after three generations of selection and subcellular localization was observed using a confocal microscope with a $488 \mathrm{~nm}$ laser (Leica SP8).

\section{Overexpression of GmTCF1a in Arabidopsis}

The GmTCF1a CDS was cloned into the XbaI and KpnI sites of the pCAMBIA1300 vector. The recombinant vector was transformed into Col-0 via Agrobacteriummediated transformation [55]. $\mathrm{T}_{0}$ generation seeds were screened with Kanamycin $(75 \mathrm{mg} / \mathrm{mL})$. Homozygous transgenic plants were obtained after three generations of Kanamycin screening. Two homozygous transgenic lines were obtained with high GmTCF1a levels.

\section{Freezing tolerance assay for the GmTCF1a overexpression plants}

A. thaliana seeds were sterilized by soaking in 50\% bleach for $5 \mathrm{~min}$, after which they were washed five times with sterile distilled water and then placed onto solidified MS medium containing $2 \%$ sucrose with the $\mathrm{pH}$ adjusted to 5.7. The 7-day-old transgenic and wildtype seedlings were transferred to pots for another 2 weeks growth $\left(21-23^{\circ} \mathrm{C}\right.$ chamber), and then treated at $4{ }^{\circ} \mathrm{C}$ for 7 days before subjected to $-8^{\circ} \mathrm{C}$ for $2.5 \mathrm{~h}$. The survival percentage of plants (number of alive plants/ total number of plants) were calculated after 7 days of recovery $\left(16: 8 \mathrm{~h}\right.$ light-dark period at $\left.22^{\circ} \mathrm{C}\right)$.

\section{Electrolyte leakage measurement}

Electrolyte leakage assays were performed with minor modifications as described by Ristic and Ashworth [57]. Briefly, three excised leaflets were placed in a $15 \mathrm{~mL}$ plastic tube containing $100 \mu \mathrm{L} \mathrm{dH}_{2} \mathrm{O}$, and then incubated in a freezing bath with $0^{\circ} \mathrm{C}$. The temperature of bath was programmed to drop to $-8^{\circ} \mathrm{C}$ in a $2^{\circ} \mathrm{C}$ reduction over $30 \mathrm{~min}$. The tubes were taken out from the bath and immediately placed on ice when the designated temperature was reached. The leaflets in $15 \mathrm{~mL}$ tubes were then transferred to $50 \mathrm{~mL}$ tubes containing $25 \mathrm{~mL}$ of $\mathrm{dH}_{2} \mathrm{O}$, the conductivity of the solution (E1) in $50 \mathrm{~mL}$ tubes was measured after shaking. The solutions' conductivities (E2) were measured again after the leaflets were autoclaved. The electrolyte leakage was calculated as the percentage of E1/E2 [27]. 


\section{Expression of cold responsive genes in GmTCF1a overexpression transgenic Arabidopsis}

Three-week-old transgenic plants were treated at $4{ }^{\circ} \mathrm{C}$ and the leaves were harvested at $0,3,6$ and $12 \mathrm{~h}$, respectively. RNA extraction and cDNA synthesis were performed as mentioned above. The specific primers for cold-responsive genes were listed in Table S1.

\section{Abbreviations}

TCF1: Tolerant to Chilling and Freezing 1; RCC1: Regulator of Chromosome Condensation Protein 1; CBF: C-repeat binding transcription factor; CRPK1: Cold-Responsive Protein Kinase 1; ICE1: Inducer of CBF Expression 1 ; bHLH: A basic Helix-Loop-Helix; COR: Cold Regulated genes; DRE: Dehydration-responsive-element; CRT: C-repeat; BCB: Blue-CopperBinding

\section{Supplementary Information}

The online version contains supplementary material available at https://doi. org/10.1186/s12870-021-03157-5.

Additional file 1: Fig. S1. Collinear genes of AtTCF1 in soybean.

Additional file 2: Fig. S2. Correlation of eight AtTCF1 homologs based on their expression levels.

Additional file 3: Fig. S3. cis-elements in the promoter region of AtTCF1 and GmTCF1s.

Additional file 4: Fig. S4. Protein sequence alignment of AtTCF1 and GmTCF1s.

Additional file 5: Fig. S5. Expression pattern of GmTCF1a.

Additional file 6: Fig. S6. Overexpression of AtTCF1 in Arabidopsis increases plant cold tolerance.

Additional file 7: Fig. S7. The relative expression level of AtCOR15a in wild-type and 35S:AtTCF1a transgenic Arabidopsis plants.

Additional file 8: Fig. S8. Domain analysis of GmTCF1s.

Additional file 9: Table S1. Primers used in this study.

\section{Acknowledgments}

Not applicable.

\section{Authors' contributions}

H.J. conceived and designed the project. Z.D. and H.W. performed the experiments and bioinformatics analysis. Z.D., X.L. and H.J. wrote the paper. All the authors read and approved the final manuscript.

\section{Funding}

This work was funded by the National Key Research and Development Program of China (2018YFD10009002). The funder was not involved in the design of the study, analysis of data, and manuscript writing.

\section{Availability of data and materials}

All data generated or analysed during the current study are available in this article and its supplementary information files. The accession numbers of AtTCF1 (At3G55580) homologs are as follows: G. max, GmTCF1a (Glyma.02G250700); GmTCF1b (Glyma.14G066000); GmTCF1c (Glyma.11G223000); GmTCF1d (Glyma.18G034600); Oryza sativa, LOC Os05g38270 and LOC Os01g62810; Medicago truncatula, Medtr3g069030; Phaseolus vulgaris, Phvul.008G230700 and Phvul.001G236500; Populus trichocarpa, Potri.008G059800 and Potri.010G199100; Linum usitatissimum, Lus10004700.g and Lus10040266.g; Gossypium raimondii, Gorai.011G268700 and Gorai.012G072600; Zea mays, GRMZM2G337819 and GRMZM2G302245; B. distachyon, Bradi2g54850; Sorghum bicolor, Sobic.003G354900 and C. sativus, Cucsa.165020.

\section{Declarations}

Ethics approval and consent to participate

Not applicable.

Consent for publication

Not applicable.

\section{Competing interests}

The authors declare that they have no competing interests.

\section{Author details}

${ }^{1}$ Shijiazhuang Academy of Agricultural and Forestry Sciences, 479 Shenglibei Street, Shijiazhuang 050041, Hebei, China. ${ }^{2}$ National Key Laboratory of Crop Genetic Improvement, College of Plant Science and Technology, Huazhong Agricultural University, Wuhan 430070, China.

Received: 17 November 2020 Accepted: 2 August 2021

Published online: 12 August 2021

\section{References}

1. Toda K, Takahashi R, Iwashina T, Hajika M. Difference in chilling-induced flavonoid profiles, antioxidant activity and chilling tolerance between soybean near-isogenic lines for the pubescence color gene. J Plant Res. 2011:124(1):173-82. https://doi.org/10.1007/s10265-010-0345-2.

2. Tyagi SK, Tripathi RP. Effect of temperature on soybean germination. Plant Soil. 1983;74(2):273-80. https://doi.org/10.1007/BF02143617.

3. Tian X, Liu Y, Huang ZG, Duan HP, Tong JH, He XL, et al. Comparative proteomic analysis of seedling leaves of cold-tolerant and -sensitive spring soybean cultivars. Mol Biol Rep. 2015;42(3):581-601. https://doi.org/10.1007/ s1 1033-014-3803-4.

4. Zhao S, Xu X, Wei D, Lin X, Qiu S, Ciampitti I, et al. Soybean yield, nutrient uptake and stoichiometry under different climate regions of Northeast China. Sci Rep. 2020;10(1):8431. https://doi.org/10.1038/s41598-020-65447-6.

5. Strauss AJ, Krüger GH, Strasser RJ, van Heerden PD. The role of low soil temperature in the inhibition of growth and PSII function during dark chilling in soybean genotypes of contrasting tolerance. Physiol Plant. 2007; 131(1):89-105. https://doi.org/10.1111/j.1399-3054.2007.00930.x.

6. Tambussi EA, Bartoli CG, Guiamet JJ, Beltrano J, Araus JL. Oxidative stress and photodamage at low temperatures in soybean (Glycine max L. Merr.) leaves. Plant Sci. 2004;167(1):19-26. https://doi.org/10.1016/j.plantsci.2004.02. 018.

7. Guy CL. Cold acclimation and freezing stress tolerance: role of protein metabolism. Annu Rev Plant Biol. 1990;41(1):187-223. https://doi.org/10.114 6/annurev.pp.41.060190.001155.

8. Hughes MA, Dunn MA. The molecular biology of plant acclimation to low temperature. J Exp Bot. 1996;47(3):291-305. https://doi.org/10.1093/jxb/4 7.3.291

9. Guo X, Liu D, Chong K. Cold signaling in plants: insights into mechanisms and regulation. J Integr Plant Biol. 2018;60(9):745-56. https://doi.org/1 $0.1111 / j i p b .12706$

10. Kaplan F, Kopka J, Sung DY, Zhao W, Popp M, Porat R, et al. Transcript and metabolite profiling during cold acclimation of Arabidopsis reveals an intricate relationship of cold-regulated gene expression with modifications in metabolite content. Plant J. 2007:50(6):967-81. https://doi.org/10.1111/j.13 65-313X.2007.03100x

11. Ormancey M, Thuleau P, Mazars C, Cotelle V. CDPKs and 14-3-3 proteins: emerging duo in signaling. Trends Plant Sci. 2017;22(3):263-72. https://doi. org/10.1016/j.tplants.2016.11.007

12. Liu Z, Jia Y, Ding Y, Shi Y, Li Z, Guo Y, et al. Plasma membrane CRPK1mediated phosphorylation of 14-3-3 proteins induces their nuclear import to fine-tune CBF signaling during cold response. Mol Cell. 2017;66(1):11728. https://doi.org/10.1016/j.molcel.2017.02.016.

13. Liu J, Shi Y, Yang S. Insights into the regulation of C-repeat binding factors in plant cold signaling. J Integr Plant Biol. 2018;60(9):780-95. https://doi. org/10.1111/jipb.12657.

14. Miura $\mathrm{K}$, Jin JB, Lee J, Yoo CY, Stirm V, Miura T, et al. SIZ1-mediated sumoylation of ICE1 controls CBF3/DREB1A expression and freezing tolerance in Arabidopsis. Plant Cell. 2007;19(4):1403-14. https://doi.org/10.11 05/tpc.106.048397. 
15. Chinnusamy V, Ohta M, Kanrar S, Lee B, Hong X, Agarwal M, et al. ICE1: a regulator of cold-induced transcriptome and freezing tolerance in Arabidopsis. Genes Dev. 2003;17(8):1043-54. https://doi.org/10.1101/gad.1 077503.

16. Ding ZT, Li C, Shi H, Wang H, Wang Y. Pattern of CS/CE1 expression under cold or drought treatment and functional verification through analysis of transgenic Arabidopsis. Genet Mol Res. 2015;14(3):11259-70. https://doi.org/1 0.4238/2015.September.22.20

17. Tang K, Zhao L, Ren Y, Yang S, Zhu JK, Zhao C. The transcription factor ICE1 functions in cold stress response by binding to the promoters of CBF and COR genes. J Integr Plant Biol. 2020;62(3):258-63. https://doi.org/10.1111/ jipb.12918.

18. Park S, Lee CM, Doherty CJ, Gilmour SJ, Kim Y, Thomashow MF. Regulation of the Arabidopsis CBF regulon by a complex low-temperature regulatory network. Plant J. 2015;82(2):193-207. https://doi.org/10.1111/tpj.12796.

19. Jia $Y$, Ding $Y$, Shi $Y$, Zhang $X$, Gong Z, Yang S. The cbfs triple mutants reveal the essential functions of CBFs in cold acclimation and allow the definition of CBF regulons in Arabidopsis. New Phytol. 2016;212(2):45-53.

20. Zhao C, Zhang Z, Xie S, Si T, Li Y, Zhu JK. Mutational evidence for the critical role of CBF transcription factors in cold acclimation in Arabidopsis. Plant Physiol. 2016;171(4):2744-59. https://doi.org/10.1104/pp.16.00533.

21. Stockinger EJ, Gilmour SJ, Thomashow MF. Arabidopsis thaliana CBF1 encodes an AP2 domain-containing transcriptional activator that binds to the C-repeat/DRE, a cis-acting DNA regulatory element that stimulates transcription in response to low temperature and water deficit. PNAS. 1997; 94(3):1035-40. https://doi.org/10.1073/pnas.94.3.1035.

22. Shi $Y$, Huang J, Sun $T$, Wang $X$, Zhu $C$, Ai $Y$, et al. The precise regulation of different COR genes by individual CBF transcription factors in Arabidopsis thaliana. J Integr Plant Biol. 2017;59(2):118-33. https://doi.org/10.1111/ jipb.12515.

23. Baker SS, Wilhelm KS, Thomashow MF. The 5'-region of Arabidopsis thaliana cor15a has cis-acting elements that confer cold-, drought- and ABAregulated gene expression. Plant Mol Biol. 1994;24(5):701-13. https://doi. org/10.1007/BF00029852.

24. Yamaguchi-Shinozaki K, Shinozaki K. A novel cis-acting element in an Arabidopsis gene is involved in responsiveness to drought, low-temperature, or high-salt stress. Plant Cell. 1994;6(2):251-64. https://doi.org/10.1105/tpc. 6.2.251.

25. Bremer A, Kent B, Hauß T, Thalhammer A, Yepuri NR, Darwish TA, et al. Intrinsically disordered stress protein COR15A resides at the membrane surface during dehydration. Biophys J. 2017;113(3):572-9. https://doi.org/1 0.1016/j.bpj.2017.06.027.

26. Li H, Ye K, Shi Y, Cheng J, Zhang X, Yang S. BZR1 positively regulates freezing tolerance via CBF-dependent and CBF-independent pathways in Arabidopsis. Mol Plant. 2017;10(4):545-59. https://doi.org/10.1016/j.molp.201 7.01.004.

27. Zhu JH, Shi H, Lee B, Damsz B, Cheng S, Stirm V, et al. An Arabidopsis homeodomain transcription factor gene, HOS9, mediates cold tolerance through a CBF-independent pathway. PNAS. 2004;101(26):9873-8. https:// doi.org/10.1073/pnas.0403166101.

28. Zhang SL, Wang YN, Li KX, Zou YM, Chen L, Li X. Identification of coldresponsive miRNAs and their target genes in nitrogen-fixing nodules of soybean. Int J Mol Sci. 2014;15(8):13596-614. https://doi.org/10.3390/ijms1 50813596

29. Xu S, Liu N, Mao W, Hu Q, Wang G, Gong Y. Identification of chillingresponsive microRNAs and their targets in vegetable soybean (Glycine max L.). Sci Rep. 2016:6:26619.

30. Yamasaki Y, Randall SK. Functionality of soybean CBF/DREB1 transcription factors. Plant Sci. 2016;246:80-90. https://doi.org/10.1016/j.plantsci.2016.02.007.

31. Chen M, Xu Z, Xia L, Li L, Cheng X, Dong J, et al. Cold-induced modulation and functional analyses of the DRE-binding transcription factor gene, GmDREB3, in soybean (Glycine max L.). J Exp Bot. 2009;60(1):121-35. https:// doi.org/10.1093/jxb/ern269.

32. Liao Y, Zou HF, Wang HW, Zhang WK, Ma B, Zhang JS, et al. Soybean GmMYB76, GmMYB92, and GmMYB177 genes confer stress tolerance in transgenic Arabidopsis plants. Cell Res. 2008;18(10):1047-60. https://doi.org/1 0.1038/cr.2008.280.

33. Liao Y, Zou HF, Wei W, Hao YJ, Tian AG, Huang J, et al. Soybean GmbZIP44, GmbZIP62 and GmbZIP78 genes function as negative regulator of ABA signaling and confer salt and freezing tolerance in transgenic Arabidopsis. Planta. 2008;228(2):225-40. https://doi.org/10.1007/s00425-008-0731-3.
34. Zhou QY, Tian AG, Zou HF, Xie ZM, Lei G, Huang J, et al. Soybean WRKYtype transcription factor genes, GmWRKY13, GmWRKY21, and GmWRKY54, confer differential tolerance to abiotic stresses in transgenic Arabidopsis plants. Plant Biotechnol J. 2008;6(5):486-503. https://doi.org/10.1111/j.14677652.2008.00336.x

35. Yu GH, Jiang LL, Ma XF, Xu ZS, Liu MM, Shan SG, et al. A soybean C2H2type zinc finger gene GmZF1 enhanced cold tolerance in transgenic Arabidopsis. PLoS One. 2014;9(10):e109399. https://doi.org/10.1371/journal. pone.0109399.

36. Ji HT, Wang YN, Cloix C, Li KX, Jenkins Gl, Wang S, et al. The Arabidopsis RCC1 family protein TCF1 regulates freezing tolerance and cold acclimation through modulating lignin biosynthesis. PLoS Genet. 2015;11(9):e1005471. https://doi.org/10.1371/journal.pgen.1005471.

37. Yamaguchi-Shinozaki K, Shinozaki K. Organization of cis-acting regulatory elements in osmotic- and cold-stress-responsive promoters. Trends Plant Sci. 2005;10(2):88-94. https://doi.org/10.1016/j.tplants.2004.12.012.

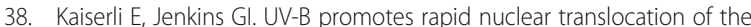
Arabidopsis UV-B specific signaling component UVR8 and activates its function in the nucleus. Plant Cell. 2007;19(8):2662-73. https://doi.org/10.11 05/tpc.107.053330.

39. Favory JJ, Stec A, Gruber H, Rizzini L, Oravecz A, Funk M, et al. Interaction of COP1 and UVR8 regulates UV-B-induced photomorphogenesis and stress acclimation in Arabidopsis. EMBO J. 2009;28(5):591-601. https:/doi.org/10.1038/emboj.2009.4.

40. Su C, Zhao H, Zhao Y, Ji H, Wang Y, Zhi L, et al. RUG3 and ATM synergistically regulate the alternative splicing of mitochondrial nad2 and the DNA damage response in Arabidopsis thaliana. Sci Rep. 2017;7(1):43897. https://doi.org/10.1038/srep43897.

41. Ji H, Wang S, Cheng C, Li R, Wang Z, Jenkins Gl, Kong F, Li X. The RCC1 family protein $\mathrm{SAB} 1$ negatively regulates $\mathrm{ABI} 5$ through multidimensional mechanisms during postgermination in Arabidopsis. New Phytol. 2019; 222(2):907-22. https://doi.org/10.1111/nph.15653.

42. Su C, Yuan J, Zhao H, Zhao Y, Ji H, Wang Y, et al. RUG3 is a negative regulator of plant responses to ABA in Arabidopsis thaliana. Plant Signal Behav. 2017;12(6):e1333217. https://doi.org/10.1080/15592324.2017.1333217.

43. Kühn K, Carrie C, Giraud E, Wang Y, Meyer EH, Narsai R, et al. The RCC1 family protein RUG3 is required for splicing of nad2 and complex I biogenesis in mitochondria of Arabidopsis thaliana. Plant J. 2011;67(6):106780. https://doi.org/10.1111/j.1365-313X.2011.04658.x.

44. Li C, Li QG, Dunwell JM, Zhang YM. Divergent evolutionary pattern of starch biosynthetic pathway genes in grasses and dicots. Mol Biol Evol. 2012; 29(10):3227-326. https://doi.org/10.1093/molbev/mss131.

45. Schmutz J, Cannon SB, Schlueter J, Ma J, Mitros T, Nelson W, et al. Genome sequence of the palaeopolyploid soybean. Nature. 2010;463(7278):178-83. https://doi.org/10.1038/nature08670.

46. Thalhammer A, Hincha DK. A mechanistic model of COR15 protein function in plant freezing tolerance: integration of structural and functional characteristics. Plant Signal Behav. 2014;9(12):e977722. https://doi.org/10.41 61/15592324.2014.977722.

47. Artus NN, Uemura M, Steponkus PL, Gilmour SJ, Lin C, Thomashow MF. Constitutive expression of the cold-regulated Arabidopsis thaliana COR15a gene affects both chloroplast and protoplast freezing tolerance. PNAS. 1996; 93(23):13404-9. https://doi.org/10.1073/pnas.93.23.13404.

48. Brown BA, Cloix C, Jiang GH, Kaiserli E, Herzyk P, Kliebenstein DJ, et al. A UVB-specific signaling component orchestrates plant UV protection. PNAS. 2005;102(50):18225-30. https://doi.org/10.1073/pnas.0507187102.

49. Yang $Y$, Liang T, Zhang L, Shao K, Gu X, Shang R, et al. UVR8 interacts with WRKY36 to regulate HY5 transcription and hypocotyl elongation in Arabidopsis. Nat Plants. 2018;4(2):98-107. https://doi.org/10.1038/s41477-017-0099-0.

50. Moura JC, Bonine CA. de Oliveira Fernandes Viana J, Dornelas M.C, Mazzafera P. abiotic and biotic stresses and changes in the lignin content and composition in plants. J Integr Plant Biol. 2010;52(4):360-76. https://doi. org/10.1111/j.1744-7909.2010.00892.x.

51. Lozano R, Hamblin MT, Prochnik S, Jannink JL. Identification and distribution of the NBS-LRR gene family in the cassava genome. BMC Genomics. 2015; 16(1):360. https://doi.org/10.1186/s12864-015-1554-9.

52. Hu B, Jin J, Guo AY, Zhang H, Luo JC, Gao G. GSDS 2.0: an upgraded gene feature visualization server. Bioinformatics. 2015;31(8):1296-7. https://doi. org/10.1093/bioinformatics/btu817.

53. Kumar S, Stecher G, Tamura K. MEGA7: molecular evolutionary genetics analysis version 7.0 for bigger datasets. Mol Biol Evol. 2016:33(7):1870-4. https://doi.org/10.1093/molbev/msw054. 
54. Bailey TL, Boden M, Buske FA, Frith M, Grant CE, Clementi L, et al. MEME SUITE: tools for motif discovery and searching. Nucleic Acids Res. 2009;37: 202-8.

55. Clough SJ, Bent AF. Floral dip: a simplified method for Agrobacteriummediated transformation of Arabidopsis thaliana. Plant J. 1998;16(6):735-43. https://doi.org/10.1046/j.1365-313x.1998.00343.x.

56. Kereszt A, Li D, Indrasumunar A, Nguyen CD, Nontachaiyapoom S, Kinkema $M$, et al. Gresshoff PM. Agrobacterium rhizogenes-mediated transformation of soybean to study root biology. Nat Protoc. 2007;2(4):948-52. https://doi. org/10.1038/nprot.2007.141.

57. Ristic Z, Ashworth EN. Changes in leaf ultrastructure and carbohydrates in Arabidopsis thaliana L.(Heyn) cv. Columbia during rapid cold acclimation. Protoplasma. 1993;172(2-4):111-23. https://doi.org/10.1007/BF01379368.

\section{Publisher's Note}

Springer Nature remains neutral with regard to jurisdictional claims in published maps and institutional affiliations.

Ready to submit your research? Choose BMC and benefit from:

- fast, convenient online submission

- thorough peer review by experienced researchers in your field

- rapid publication on acceptance

- support for research data, including large and complex data types

- gold Open Access which fosters wider collaboration and increased citations

- maximum visibility for your research: over $100 \mathrm{M}$ website views per year

At BMC, research is always in progress.

Learn more biomedcentral.com/submissions 